\title{
Pasivas de verbos sicológicos inacusativos
}

\author{
Rafael Camacho Ramírez \\ Universidad Nacional Mayor de San Marcos \\ elrafaelcamacho@gmail.com
}

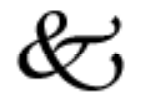

Resumen

El presente trabajo plantea la posibilidad de analizar oraciones como María se aburrió de hacer lo mismo como pasivas pronominales, derivadas de las versiones inacusativas de estos verbos sicológicos: A María le aburrió hacer lo mismo. Fundamentalmente, las pruebas que se presentan son de carácter semántico, es decir, tanto la versión pasiva como la inacusativa presentan un significado análogo, específicamente, presentan el rasgo semántico de Causa interna. Las versiones causativa y agentiva del verbo sicológico quedan excluidas como input por carecer de este rasgo. El mecanismo sintáctico que permite formar pasivas a partir de verbos que no tienen argumento externo, por ejemplo los inacusativos, es el desarrollado por Baker (1988), y es el que usaremos en este trabajo.

Palabras claves: Verbo sicológico, verbo causativo, verbo inacusativo, pasiva, causa interna, permanencia.

\begin{abstract}
The present work presents the possibility to examine sentences like María se aburrió de hacer lo mismo as pronominal passive, derived of the unaccusative versions of these psychological verbs: A María le aburrió hacer lo mismo. Fundamentally the evidence that I present is of semantic character, that is, so much the passives versions like the unaccusative present a similar meaning, specifically, they present internal Cause's semantic feature. The causative versions and agentive of the psychological verb are excluded like input to lack this feature. The syntactic mechanism that it allows to forming passive as from verbs that they do not have external argument, for example the unaccusatives, is what baker develops (1988), and it is the one that we will use in this work.
\end{abstract}

Keywords: psychological verb, causative verb, unaccusative verb, passive, internal Cause, permanence. 


\section{El problema de investigación}

En general, el tema de los verbos sicológicos en nuestra lengua no ha sido muy estudiado. En la tradición generativa, lo que ha interesado, sobre todo, son los aspectos sintácticos de estos verbos, es decir, la diferente distribución de los roles de un mismo verbo (Belletti y Rizzi 1987, Grimshaw 1990, entre otros).

En este trabajo se postula el mecanismo de pasivización para explicar parte de esa variada distribución. Proponer este mecanismo sintáctico supone demostrar que es posible pasivizar en nuestra lengua verbos que no tienen rol externo; aunque éste es un fenómeno que ocurre en algunas lenguas (Baker 1988), nunca se postuló para el castellano.

Un aspecto central en el trabajo es el de poner en relación una estructura con otra, en una especie de cadena derivativa hasta llegar a la pasiva. Esta cadena supone una aproximación semántica detallada a todas las versiones posibles de los verbos sicológicos.

\section{Marco teórico}

El presente trabajo se enmarca dentro de la Teoría de Principios y Parámetros, específicamente el modelo de Principios y Parámetros (Chomsky 1981). En este modelo se parte del hecho de que existen principios universales innatos que conforman el desarrollo de una lengua. Además de estos principios, la gramática interiorizada cuenta con parámetros, que explican las variaciones entre las lenguas. Lo que sigue es un esquema que pretende mostrar el lugar en el que los principios operan: 


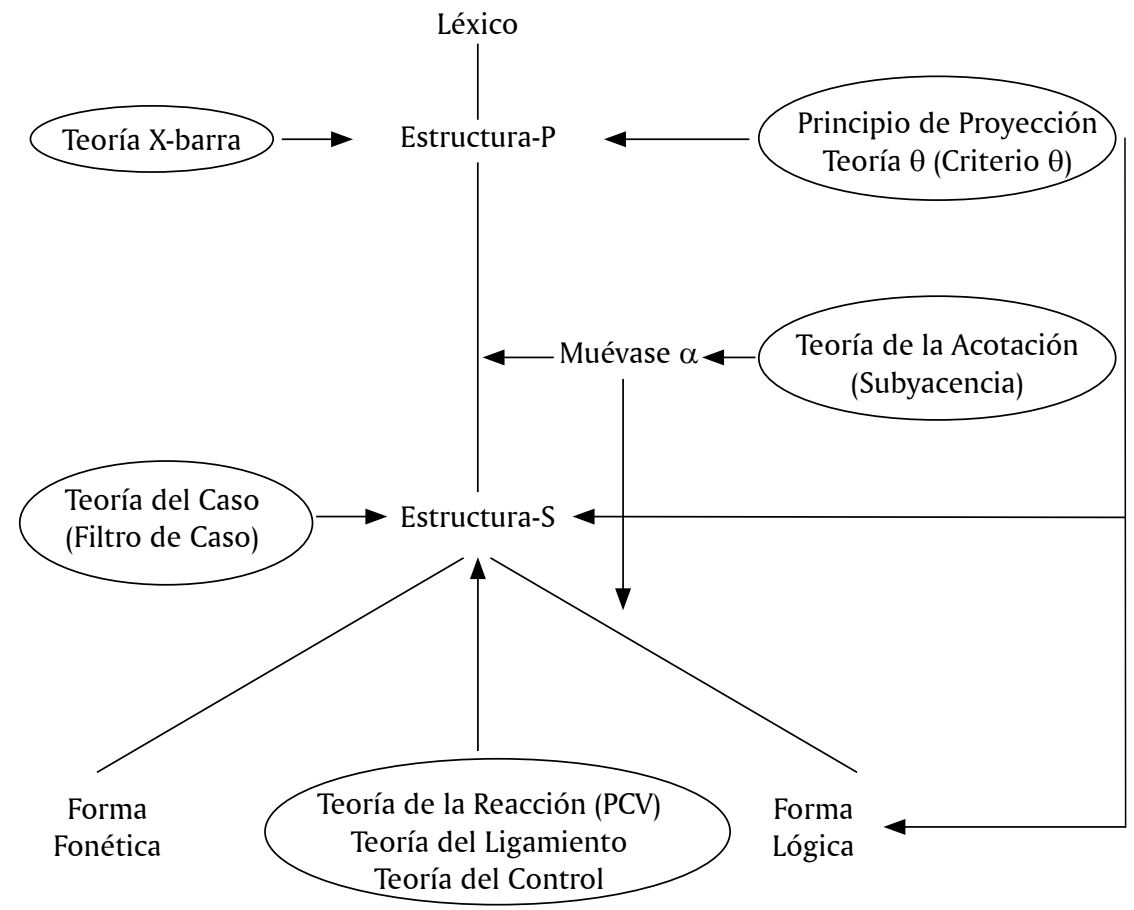

Quisiera definir algunos conceptos que irán apareciendo a lo largo del trabajo:

a) Esta lista de roles y su definición están basadas en Saeed (1998):

Agente: el iniciador de alguna acción, capaz de actuar con volición.

Paciente: la entidad que sufre el efecto de alguna acción, frecuentemente sufre algún cambio en su estado.

Tema: la entidad que es movida por alguna acción, o cuya locación es descrita.

Experimentante: la entidad que es consciente de la acción o estado descrito por el predicado, pero que no está en control de esa acción o estado. 
Beneficiario: la entidad por cuyo beneficio la acción fue hecha. Instrumento: el medio por el que una acción es hecha.

Por lo general, se entiende que la diferencia entre Causa y Agencia es la volición. Así, en 1 uno puede pensar que el sujeto no es Agente sino Causa:

1) El aire abrió la puerta.

Algunas veces se ha presentado la diferencia como una entre los rasgos animado e inanimado; pero como veremos a lo largo de este trabajo, no es necesariamente así. El rol Causa es aquel que no tiene intención o voluntad de hacer lo que indica el verbo. Así en la oración 2, si Juan es Agente tendrá toda la intención de preocupar a Elena, tal vez contándole algo que sabe lo hará; sin embargo, si interpreto a Juan como Causa, él no será consciente de que, por ejemplo, el hecho de hablar solo preocupará a Elena:

2) Juan (la) preocupa a Elena.

b) Incorporación

El concepto de incorporación manejado por Baker (1988) es un caso del movimiento de núcleo a núcleo, que a su vez es un subcaso de Muévase-alfa.

La restricción sobre el movimiento de los núcleos está expresada de la siguiente manera:

Un $\mathrm{X}^{0}$ sólo puede moverse a un $\mathrm{Y}^{0}$ (que lo rija propiamente) Travis (1984:131), citado en Demonte (1989)

Con el concepto de Incorporación, Baker puede explicar, por ejemplo, lo que sucede en el cambio de las funciones gramaticales de una antipasiva. Esta construcción ha sido descrita como una en la que un morfema es añadido a un verbo transitivo, lo que ocasiona que el objeto directo del verbo termine en una frase oblicua. El autor menciona, entre otros, el siguiente caso:
a. Angut-ip
arnaq
unatar-paa.
Man-ERG woman (ABS) beat-INDIC:3sS/ 3sO 
The man beat the woman.

(Greenlandic Eskimo; Sadock (1980))
b. Angut
arna-mik unata-a-voq.
Man (ABS) woman-INSTR beat-APASS-INDIC:3sS
The man beat the woman.

El morfema antipasivo A que se une al verbo (unata) en b. hace que el objeto termine en una frase instrumental.

La explicación de Baker es que el morfema antipasivo se incorpora al verbo, respetando el Principio de la Categoría vacía. Se trata de un caso de incorporación de un nombre ya que el morfema antipasivo se comporta como tal al recibir rol y Caso del verbo. Algo muy similar ocurre con el mecanismo de pasiva que manejamos aquí ${ }^{1}$.

\section{c) Causa interna}

Tomaré las ideas desarrolladas en Mendikoetxea (1999) para definir este concepto. Basándose en Levin y Rappaport (1995), la autora señala que un "predicado expresa una eventualidad de Causa interna cuando existe una propiedad inherente al único argumento del verbo que es responsable de que se realice el evento que denota el predicado" (1597). En verbos como Preocupar, en su uso inacusativo, habría tal Causa interna:

1) A Juan le preocupa el clima actual.

Aunque tenemos dos argumentos, podemos decir que al Experimentante tiene una propiedad inherente responsable de que se realice lo que dice el verbo. Lo que dice la oración es que el Tema de su preocupación es el clima, es decir, que la información que él tiene lo lleva a concluir que el clima actual es preocupante. Aunque es posible imaginar que el clima puede actuar como la causa directa de su preocupación, no es eso lo que dice 1. La Causa interna sería esa información que el Exp. lleva consigo, y que coincide con el Tema en la experiencia que describe el verbo. Lo que vemos no es el desencadenamiento

1 Véase la página 14. 
de una experiencia provocada por una Causa externa sino una Coincidencia entre lo que el Exp. lleva en sí y un Tema.

Los ejemplos que siguen son casos de verbos con Causa interna:

1) María palideció.

2) Pedro adelgazó.

3) El rosal floreció.

El sujeto sufre el cambio de estado que denota el predicado, de modo que se puede considerar como el objeto lógico o semántico. Es esto lo que vemos en 1. El Exp. es el objeto lógico del verbo, que sufre lo que indica éste.

Señala la autora que los verbos de Causa interna pueden pertenecer a la clase de los inergativos (Jugar, Hablar, por ejemplo) o a la de los inacusativos (Palidecer, Caer, por ejemplo). Verbos sicológicos como Preocupar en sus versiones dativas serían casos de verbos inacusativos (Belletti y Rizzi 1987).

\section{d) Permanencia}

Continuidad de la Causa interna, responsable de la experiencia descrita por el verbo.

\section{Hipótesis}

La hipótesis central del trabajo es la siguiente:

Las versiones pronominales de verbos sicológicos como Preocuparse serían casos de pasivas derivadas de verbos inacusativos,

Una segunda hipótesis es:

Es posible establecer semejanzas y diferencias en el significado del rol Experimentante de los verbos sicológicos gracias a la presencia del rol Causa interna.

\section{Análisis}

Las oraciones a y b serían el resultado de un proceso de pasivización que ha aplicado sobre verbos sicológicos inacusativos: 
a) Elena se aburrió de ver la película.

b) Juan se preocupa de la situación.

Observemos las siguientes oraciones:

1) Ver la salida del sol (la) aburrió a Elena.

2) A Elena le aburrió ver la salida del sol.

3) Elena se aburrió de ver la salida del Sol.

En la oración 1, el sujeto tiene el rol Causa externa-Tema ${ }^{2}$, y el OD es el Exp. El hecho que se describe en 1 ocurre en un momento determinado, es decir, en un punto específico en el tiempo. En la segunda oración, el rol Causa externa se ha perdido; ahora el sujeto es solo Tema. Como consecuencia de esto, no es algo externo lo que desencadena la experiencia. El responsable parece ser el Exp. mismo. Algo interno en él provoca la experiencia que sufre. Llamaré a este desencadenante Causa interna (CI). Mendikoetxea (1999) define la CI así: Un predicado expresa una eventualidad de Causa interna cuando existe una propiedad inherente al único argumento del verbo que es responsable de que se realice el evento que denota el predicado. De otro lado, que la inacusativa decausativice o detransitivice, es decir, que borre la Causa externa es un tópico común. Por ejemplo, el verbo Empeorar tiene dos versiones una transitiva o causativa (Juan empeoró la situación) y otra sin Causa, inacusativa (La situación empeoró). Otros verbos con esta alternancia son: desaparecer, cicatrizar, despertar, hervir, etc.

Un efecto de la presencia de la $\mathrm{Cl}$ en 2 es que el hecho que expresa el verbo perdió la especificidad temporal, es decir, podemos interpretar en 2 que la experiencia de algún modo continúa. Incluso es posible interpretar en 2 que Elena tomó la decisión de ya no ver más la salida del Sol; lo que de algún modo "perenniza" la experiencia sufrida. Es muy difícil interpretar esta decisión en 1.

En la tercera oración tenemos los mismos roles que en la segunda, aunque en este caso el Exp. es el sujeto y el Tema es la FP. Me

2 Se trata de una Causa externa ya que no está en el Experimentante 
parece más claro que en la oración 3 la experiencia depende enteramente del Exp., y no de una Causa externa, es decir, se puede notar un mayor control o participación del Exp. en la experiencia. Ahora, si comparamos la participación del Exp. en las tres oraciones, notamos que en la causativa 1, el Exp. prácticamente no tiene ninguna participación activa, dicho de otro modo, él sufre irremediablemente la experiencia; contrariamente, en la inacusativa y pasiva la experiencia depende de la actividad del propio Exp., aunque esta actividad se hace más evidente en la versión con Se. Si el análisis es correcto, entonces la versión pronominal del verbo enfatizaría o remarcaría el hecho de que la experiencia depende, en última instancia, de algo interno al Exp., y no de una fuerza externa.

En la versión inacusativa es posible, según lo establecido, interpretar que la experiencia de algún modo sigue vigente; un rasgo que la causativa no mostraba. Ampliemos un poco más este asunto.

Observemos las siguientes oraciones:

4) A María le gustó la película.

5) A Sandra le fascinó la música.

En la inacusativa 4 podemos interpretar que la película no ha dejado de gustarle a María, es decir, la experiencia ocurrió en el pasado, pero no se quedó ahí. Compárese con la oración Juan golpeó la puerta, en esta oración la acción se dio en el pasado y se quedó ahí. Podemos decir entonces que en la inacusativa hay un efecto de Permanencia de la experiencia ${ }^{3}$. De modo distinto, en la causativa 1 el desencadenamiento de la experiencia, su inicio y su fin, ocurre, como decíamos, en un momento específico, en el momento en el que la Causa externa incide sobre el Exp., o dicho de otro modo, el momento en el que el Exp. sufre la acción de la Causa externa. No encontramos un momento final específico en la inacusativa ${ }^{4}$ porque

3 Este efecto lo notamos también en la inacusativa 2, así, podemos pensar que no ha dejado de aburrirle ver la salida del Sol.

4 El momento inicial de la experiencia puede identificarse con el momento en el que el Exp. toma contacto con el Tema. En el caso de 4, con el momento en el que María vio la película; 
la CI siempre estaría presente en el Exp. La CI es una propiedad inherente que, en este caso, cuando se conecta con el Tema adecuado, genera la experiencia; ahora, si la $\mathrm{CI}$ permanece, entonces la experiencia provocada por ella, también lo hará. Si es correcto el análisis, entonces el rasgo Permanencia puede ser explicado por la presencia de la $\mathrm{CI}$.

En la oración 3 -una pasiva según nuestra hipótesis-, también notamos Permanencia. Podemos interpretar que el Exp. no ha cambiado de opinión respecto del Tema, es decir, sigue pensando lo mismo respecto de ver la salida del Sol. De modo que podemos establecer que inacusativa y pasiva presentan el rasgo Permanencia.

Quisiera aclarar que no podría ser la experiencia en sí lo que permanece precisamente; el aburrimiento o el gusto, en 2, 3 y 4 respectivamente, no ocurren una y otra vez indefinidamente; han ocurrido en momentos específicos. Podemos preguntarnos qué es lo que permanece en el Exp. entonces. Permítaseme presentar cómo entiendo el funcionamiento de la $\mathrm{Cl}$ : Las experiencias son provocadas por un juicio que el Exp. hace sobre el Tema. Este juicio se sostiene necesariamente en la presencia de cierta información que yace en el Exp.; esta información sería la CI. Entonces, la información del Exp., la CI, al entrar en contacto con el Tema genera un juicio, que es lo que lleva a la experiencia. Si la información, la CI, no cambia, entonces el juicio será permanente y la experiencia también. De modo que lo que realmente permanece no es la experiencia sino la opinión, el juicio que la sostiene. Esto es lo que interpretaríamos como permanente en 2, 3, 4 y 5 .

En lo que sigue trataré de mostrar que la pasiva deriva de la inacusativa, porque comparte el rasgo de Permanencia con ella. Se excluye así a la versión causativa como posible "fuente", ya que no presenta Permanencia.

Observemos las siguientes oraciones:

sin embargo, no hay un momento final ya que la experiencia, según nuestra interpretación, permanece en el Exp. 
7) Juan aburría a Sonia cada vez que se encontraban.

8) A Sonia le aburría Juan cada vez que se encontraban.*

9) Sonia se aburría de Juan cada vez que se encontraban.*

En la oración 6, la experiencia dura lo que dura el encuentro. En la oración 7, el adjunto no es permitido porque la experiencia en el Exp. es permanente debido a la $\mathrm{Cl}$; sin embargo, el adjunto trata de introducir un momento específico de ocurrencia, a saber, que la experiencia, el aburrimiento, aparecía solo cada vez que se encontraban. La versión pronominal 8 tampoco permite el adjunto, creo yo, por las mismas razones.

Observemos el siguiente grupo:

9) Juan preocupó a María en la reunión.

10) A María le preocupó Juan en la reunión.*

11) María se preocupó de Juan en la reunión.*

En 9 el adjunto delimita el tiempo en el que se dio la reacción ${ }^{5}$. En 10 y 11 este límite torna agramaticales a las oraciones.

12) Juan preocupó adrede a María con sus historias.

La oración 12 es una agentiva ${ }^{6}$. El sujeto es Agente, pero no Tema; el OD es el Exp. y la FP presenta el Tema de la preocupación. Esta FP es un adjunto; no es un argumento del verbo. La inacusativización de un verbo operaría sobre los argumentos del verbo, no sobre adjuntos. Entonces, no podría obtener una inacusativa de una agentiva como 12 sino de una versión causativa como 9, ya que en esta oración el rol Tema sí forma parte de los argumentos del verbo: aparece unida a la Causa externa en el sujeto. El verbo Interesar parece probar lo dicho:

5 Debemos interpretar en esta oración que Juan no tuvo la intención de preocupar a María; Juan tiene el rol Causa-Tema, no el de Agente. Así, podemos imaginar que Juan estaba hablando solo y no fue consciente de que era observado por María; entonces Juan es el asunto de la preocupación de María y el desencadenante de ésta, aunque sin intención.

6 Aquí Juan sí tiene la intención de preocupar a María 
13) Armando interesó a los chinos en el negocio.

14) A los chinos les interesó el negocio.

15) Los chinos se interesaron en el negocio.

16) El negocio los interesó."

La oración 13 es una agentiva; el verbo no permite causativa, véase la oración 16. Pues la inacusativa 14 debe derivar necesariamente de la agentiva. Creo que esto es posible porque el Tema, en Interesar, sí es argumento del verbo, a diferencia de 12 . De modo que no importa si la fuente para la inacusativa es causativa o agentiva. Lo que parece importar es que el rol Tema sea parte de los argumentos del verbo.

Hemos llegado a establecer que la única "fuente" (input) posible para una inacusativa es la causativa. Hemos descartado a la versión agentiva como posible fuente. Sigamos con algunos verbos más que muestran que, en la cadena derivativa que establecemos, inacusativa y pasiva presentan el rasgo común de Permanencia.

El verbo Alegrar muestra también la presencia de Permanencia en inacusativas y pasivas:

17) Ayer la solidaridad de la gente lo alegró.

18) Ayer a Sergio le alegró la solidaridad de la gente.ii

19) Sergio se alegró ayer de la solidaridad de la gente.i¿

El adjunto ayer no es posible en la inacusativa 18 ni pasiva 19 porque delimita el tiempo en un verbo con Permanencia. En la versión causativa 17, el adjunto sí es aceptado porque esta presentación del verbo no tiene Permanencia. Algo similar ocurriría en 23, 24 y 25 . El adjunto inmediatamente tampoco es permitido en 21 y 22 , aunque sí en la versión causativa 20 por iguales razones:

20) Verlo la alegra inmediatamente.

21) A Silvia le alegra inmediatamente verlo.ii

22) Silvia se alegra inmediatamente de verlo.ii 
23) La actitud de Juan (la) avergonzó ayer a María.

24) Ayer a María le avergonzó la actitud de Juan.ic

25) Ayer María se avergonzó de la actitud de Juan.i¿

Veamos ahora el particular caso del verbo Enamorar. Observemos el siguiente grupo de oraciones:

26) Juan enamoró a María.

27) María se enamoró de Juan.

28) María fue enamorada por Juan.

29) A María le enamoró Juan.*

En 26 el sujeto es Agente y Tema a la vez. El verbo no tiene inacusativa (29). ¿Cómo es posible ir directamente de la agentiva a la pasiva? Pues, si no media una inacusativa es porque el Exp. debe tener ya $\mathrm{CI}$.

Podemos establecer, respecto de la cadena derivativa que defendemos aquí, que no podemos saltar de una transitiva-causativa a una pasiva porque la primera tiene Causa externa y la pasiva requiere de Causa interna. De modo que la inacusativa puede ser vista como el paso necesario para que haga su aparición la $\mathrm{CI}$. Ahora, es de suponer que si la transitiva presenta ya $\mathrm{CI}$, podremos ir entonces directamente a la pasiva, sin necesidad de pasar por la inacusativa. Creo que esto es lo que ocurre en el verbo Enamorar. Efectivamente, podemos interpretar en 26 Permanencia, podemos interpretar que María sigue enamorada de Juan aun cuando la acción está en pasado. Si hay Permanencia en el Exp. es porque este tiene CI. Considero que es el Agente el responsable de que María tenga CI. Este Agente busca ser amado por el Exp., esto es, busca crear en el Exp. una opinión favorable hacia él (o crear los elementos que lleven a esta opinión), en otras palabras, busca una $\mathrm{CI}$ favorable hacia él. Si el verbo en 26 expresa la realización del evento, entonces ese Exp. tiene $\mathrm{CI}$, y podemos ir directamente a la pasiva 27.

La pasiva participial 28 parece referirse sólo al evento en el cual el 
agente hace lo suyo; pero no a la actividad del Exp., a esto se refiere la versión con Se 27.

\section{Algunas restricciones}

30) María molesta a Juan.

31) A Juan le molesta María.i¿

32) Juan se molesta de María.*

El sujeto de 30 no puede ser interpretado sino como Agente. No puede ser interpretado como Causa porque todo él no puede ser el Tema de la molestia; de otro lado, es muy difícil imaginar qué aspecto de él pueda serlo ${ }^{7}$. Al no tener versión causativa no puedo derivar inacusativa (31) ni pasiva (32). Sin embargo, si cambiamos a un sujeto inanimado, sí podemos tener el rol Causa-Tema, y también la derivación:

33) La irresponsabilidad de Juan molesta a María.

34) A María le molesta la irresponsabilidad de Juan.

35) María se molesta de la irresponsabilidad de Juan.

Ahora, es posible una oración como 36 con un sujeto animado:

36) María se molestó con Juan.

La oración 36 parece indicar sobre todo la reacción a la molestia. Así, uno puede pensar, a partir de 36, que ella empezó a evitar a Juan o alguna otra reacción similar. Hay algo más que la molestia en sí. Es una versión bastante activa del verbo. En esta versión la restricción del tipo de FN desaparecería. Lo mismo sucedería con Enojar:

37) Rodrigo/todo enoja a Marta.

38) A Marta le enoja Rodrigo.ii/ todo.

7 Con Aburrir o Preocupar sí es posible pensar en algún aspecto que pueda desencadenar la experiencia. Véase las oraciones 6 y 9 , respectivamente. 
39) Marta se enoja de Rodrigo.*/ todo.

40) Marta se enoja con Rodrigo.

Ahora veamos algunas pruebas que muestran la presencia de $\mathrm{CI}$ en inacusativas y pasivas:

41) La actitud de Mirta lo decepcionó justificadamente.*

42) A Juan le decepcionó justificadamente la actitud de Mirta.

43) Juan se decepcionó justificadamente de la actitud de Mirta.

44) La justificación lo sorprendió inexplicablemente.*

45) A Juan le sorprendió inexplicablemente la justificación.

46) Juan se sorprendió inexplicablemente de la justificación.

47) Que Mirta haya conseguido trabajo alegró hipócritamente a Juan.ic

48) A Juan le alegró hipócritamente que Mirta haya conseguido trabajo.

49) Juan se alegró hipócritamente de que Mirta haya conseguido trabajo.

Todos los adjuntos son posibles en la inacusativa y pasiva, pero no en la causativa. Esto sería debido a que tales adjuntos implican actividad en el Exp., y tal actividad solo parece posible en los Exp. con $\mathrm{CI}$, es decir, en los Exp. de la inacusativa y pasiva.

Hasta aquí he tratado de poner en relación a inacusativas y pasivas de acuerdo con el rasgo semántico Permanencia, el que sería compartido por estas dos construcciones.

Hay verbos sicológicos con el Exp. como sujeto profundo. Es el caso de Gustar. Veamos cómo funciona la derivación con este verbo:

50) Juan gusta de las bromas.

51) A Juan le gustan las bromas.

52) Juan se gusta de las bromas.*

Según Cuervo (2002), Gustar de es la primera versión de este verbo. En la oración 50 interpretamos que a Juan le gusta hacer bromas 
o escucharlas. En 51 esa posibilidad no existe, solo dice que le gustan las bromas en sí. La inacusativa ha borrado el hacer. Lo mismo vimos en la derivación de los causativos Aburrir o Preocupar; la versión inacusativa borraba la Causa externa. Podemos decir entonces que la inacusativa, en verbos sicológicos, focaliza lo que sucede en el Exp., borrando cualquier tipo de actividad o hacer, es decir, produce una decausativización, como mencionábamos al inicio. La pasiva 52 no es posible porque sería innecesaria. El énfasis de la actividad del Exp. que hace la pasiva no sería necesario ya que es bastante claro que los gustos dependen siempre del Exp. y nunca de algo externo, de modo que enfatizar la ausencia de una Causa externa sería redundante. Siendo así, la oración 52 terminaría diciendo algo muy similar a 51. En el caso de las versiones causativas de verbos como Aburrir o Preocupar, no es redundante la versión pasiva ya que el verbo tiene un antecedente con Causa externa; pero este no es el caso de Gustar.

Observemos el comportamiento del siguiente peculiar grupo:

53) Su voz todo lo encantaba.

54) La mentira lo fascina.

55) El vino lo agrada."

56) A María le encanta el vino.

57) A Juan le fascina la música.

58) A Elena le agrada viajar.

59) Juan se encantó con la belleza de María.

60) Juan se fascinó con la sabiduría de Elena.

61) Elena se agrada de saber la respuesta.

53 y 54 no son comunes, son marginales. 55 es del todo agramatical. Las versiones inacusativas son buenas todas. Las versiones con Se no son comunes ni registradas, aunque no son del todo rechazadas por los hablantes consultados.

Creo que, en general, a estos tres últimos verbos (encantar, fascinar y agradar) se les interpreta como que todo depende del Exp. Si es así, entonces la inacusativa es su lugar natural. La pasiva se generaría, como decía al inicio, para enfatizar el hecho de que todo 
depende del Exp. Hay la necesidad de enfatizar cuando el verbo tiene antecedentes causativos y agentivos; pero con estos tres verbos, no parece haber tales antecedentes; los usos transitivos son muy marginales.

\section{Mecanismo Sintáctico}

En general, las pasivas de inacusativos son consideradas imposibles de realizarse, porque el verbo no tiene rol externo. Como consecuencia de esto, el morfema pasivo se quedaría sin rol$^{8}$. Sin embargo, según Baker, hay dos clases de morfemas pasivos: uno que es categorialmente INFL y otro que es un Nombre. El morfema -do del castellano, al igual que el morfema -en del inglés, es INFL ${ }^{9}$, por lo que solo podrá recibir el rol externo del verbo, por lo tanto, no habrá pasivas participiales de verbos inacusativos:

62) Juan fue aburrido por/de ver la salida del Sol.*

63) Juan fue preocupado por/de la situación."

1)

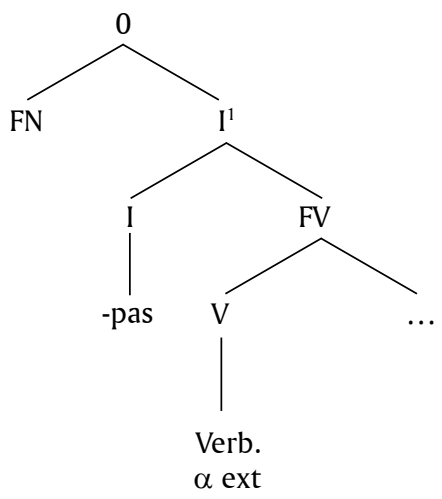

8 Se asume, en general, que el morfema pasivo toma el rol externo del verbo. Por ejemplo, véase Jaeggli (1986).

9 El diagrama 1 muestra la forma cómo es la asignación de rol al morfema INFL. 
Sin embargo, el morfema Se del castellano sería un pasivizador Nombre, por lo tanto, puede aparecer en cualquier posición, incluso al interior de la FV, en donde podrá recibir un rol interno del verbo. Con el Se, entonces, es posible tener pasivas de verbos que no tienen rol externo, verbos como los inacusativos.

En el diagrama 2, el morfema Se ha tomado el rol que le correspondía al Tema, por lo que debe aparecer la preposición De para darle un rol a la FN. El Se también ha tomado el Caso que le correspondía al Exp., por lo tanto, este debe viajar a la posición sujeto para recibir ahí su Caso. Luego el morfema Se se clitizaría al verbo, en un proceso que Baker denomina Incorporación, que es un caso del movimiento de núcleo a núcleo.

2)

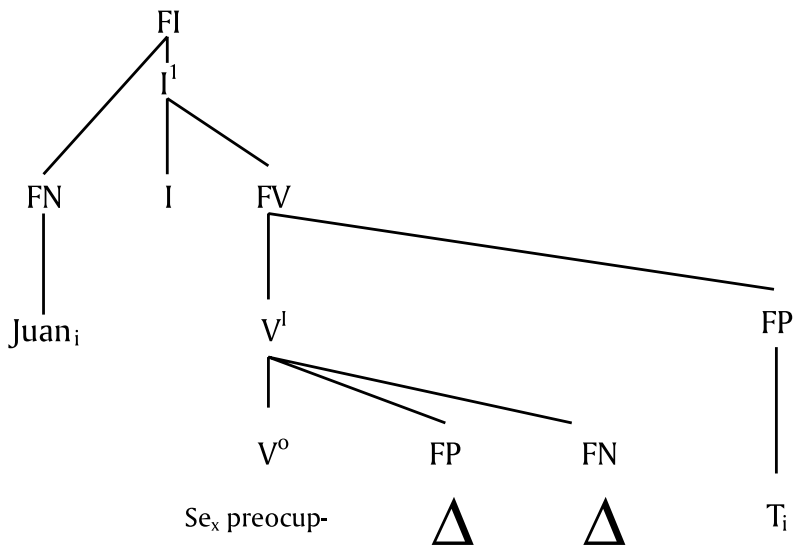

\section{Conclusiones}

1. En el castellano habría dos morfemas pasivos de distinta categoría: -do es un INFL y Se es un nombre. Esta distinción permite prever la posibilidad de tener pasivas de verbos que no tienen argumento externo, como los inacusativos.

2. Las FN con rol Experimentante pueden tener Causa interna.

3. Un verbo sicológico como Aburrir o Preocupar puede llegar a tener cuatro versiones semánticas distintas: agentiva, causativa, inacusativa y pronominal. 
4. En el castellano sería posible tener pasivas pronominales de verbos inacusativos sicológicos. El rasgo semántico Causa interna sería el vínculo entre la versión inacusativa y la pasivización de ésta, la versión con Se.

5. El objetivo de la pasivización pronominal en estos verbos sería enfatizar la Causa interna del Experimentante de la oración inacusativa.

\section{Referencias bibliográficas}

Baker, Mark (1988): Incorporation, A Theory of Grammatical function Changing. Chicago, Chicago University Press.

Belletti, Adriana, y Rizzi, Luigi (1987): "Los Verbos Psicológicos y la Teoría Temática”. En: Demonte, V. y Fernández Lagunilla, M. (eds.)

\section{Arquero.}

(1987): Sintaxis de las Lenguas Románicas. Madrid, El

Cuervo, Rufino (2002): Diccionario de Construcción y Régimen de la Lengua Castellana. Barcelona, Herder.

Chomsky, Noam (1981): Lectures on Government and Binding. Cambridge, MA, The MIT Press.

Demonte, Violeta (1989): Teoría Sintáctica. De las estructuras a la rección. Madrid, Síntesis.

Grimshaw, Jane (1990): Argument Structure. Cambridge, MA, The MIT Press.

Jaeggli, Osvaldo (1986): "Passive”. Linguistic Inquiry, 17; 587-622.

Mendikoetxea, Amaya (1999): "Construcciones Inacusativas y Pasivas”. En: BOSQUE, Ignacio y DEMONTE, Violeta (dir.): Gramática Descriptiva de la Lengua Española, Tomo II, Madrid, Espasa Calpe; pp.1575-1630.

Real Academia Española (2001): Diccionario de la Lengua Española. Vigésima segunda edición. Madrid, Espasa Calpe.

Saeed, John (1997): Semantics. Massachussets, Blackwell Publishers. 